\title{
Video Article \\ Modeling Biological Membranes with Circuit Boards and Measuring Electrical Signals in Axons: Student Laboratory Exercises
}

\author{
Martha M. Robinson ${ }^{1}$, Jonathan M. Martin ${ }^{1}$, Harold L. Atwood ${ }^{2}$, Robin L. Cooper ${ }^{1}$ \\ ${ }^{1}$ Department of Biology, University of Kentucky \\ ${ }^{2}$ Department of Physiology, University of Toronto \\ Correspondence to: Robin L. Cooper at RLCOOP1@email.uky.edu
}

URL: https://www.jove.com/video/2325

DOI: doi:10.3791/2325

Keywords: Basic Protocols, Issue 47, Invertebrate, Crayfish, Modeling, Student laboratory, Nerve cord

Date Published: 1/18/2011

Citation: Robinson, M.M., Martin, J.M., Atwood, H.L., Cooper, R.L. Modeling Biological Membranes with Circuit Boards and Measuring Electrical Signals in Axons: Student Laboratory Exercises. J. Vis. Exp. (47), e2325, doi:10.3791/2325 (2011).

\section{Abstract}

This is a demonstration of how electrical models can be used to characterize biological membranes. This exercise also introduces biophysical terminology used in electrophysiology. The same equipment is used in the membrane model as on live preparations. Some properties of an isolated nerve cord are investigated: nerve action potentials, recruitment of neurons, and responsiveness of the nerve cord to environmental factors.

\section{Video Link}

The video component of this article can be found at https://www.jove.com/video/2325/

\section{Protocol}

\section{INTRODUCTION}

\section{1) Background}

A fundamental knowledge of electrical circuits is a valuable tool for understanding and conceptualizing many aspects of physiological experimentation and theory. This exercise is intended to acquaint you with some general principles involving voltage sources, electrical resistance, and electrical capacitance. These introductory concepts provide the background for understanding such phenomena as synaptic transmission and the spread of electrical signals along a nerve fiber.

A word of caution: In standard everyday electrical circuits composed of metal wires and resistors, CURRENT is carried by electrons, which have a negative charge. In biological systems, current is carried by IONS, which may have one or more positive or negative charges. By convention, current flows from positive to negative (although of course electrons actually move the other way).

The components of the circuit represent the electrical properties of living tissue and in some cases that of inanimate objects (such as electrodes) involved in its study.

In working through the exercise, keep notes of results and calculations.

\section{2) General Principles}

The basic properties of electrical circuits in which direct current is flowing are described by Ohm's Law

$V=I R$

Where $\mathrm{V}=$ voltage in volts $(\mathrm{V})$

$I=$ current in amps $(A)$

$\mathrm{R}=$ resistance in ohms $(\Omega)$

This law governs the potentials which are observed in nerve cells with electrodes. We will first demonstrate Ohm's Law and its application in biological materials.

1.3) Materials

\begin{tabular}{|l|l|}
\hline Item & Quantity \\
\hline Breadboard & 1 \\
\hline
\end{tabular}




\begin{tabular}{|c|c|}
\hline Resistors: $330 \mathrm{~K} \Omega$ & 1 \\
\hline$: 100 \mathrm{~K} \Omega$ & 12 \\
\hline$: 33 \mathrm{~K} \Omega$ & 6 \\
\hline$: 22 \mathrm{~K} \Omega$ & 1 \\
\hline$: 10 \mathrm{~K} \Omega$ & 6 \\
\hline$: 4.7 \mathrm{~K} \Omega$ & 1 \\
\hline$: 2.2 \mathrm{~K} \Omega$ & 1 \\
\hline$: 1.5 \mathrm{~K} \Omega$ & 1 \\
\hline$: 1 \mathrm{~K} \Omega$ & 1 \\
\hline$: 510 \Omega$ & 1 \\
\hline$: 330 \Omega$ & 1 \\
\hline$: 220 \Omega$ & 1 \\
\hline$: 150 \Omega$ & 1 \\
\hline$: 100 \Omega$ & 1 \\
\hline Capacitors: $0.1 \mu \mathrm{F}$ & 6 \\
\hline$: 10 \mu \mathrm{F}$ & 6 \\
\hline Wires & 12 \\
\hline PowerLab & 1 \\
\hline PowerLab Output Leads & 1 \\
\hline PowerLab Input Leads & 1 \\
\hline Voltmeter (Current Capable) & 1 \\
\hline
\end{tabular}

\section{Resistor Coding Key}

\begin{tabular}{|l|l|}
\hline Color & Digit \\
\hline Black & 0 \\
\hline Brown & 1 \\
\hline Red & 2 \\
\hline Orange & 3 \\
\hline Yellow & 4 \\
\hline Green & 5 \\
\hline Blue & 6 \\
\hline Violet & 7 \\
\hline Grey & 8 \\
\hline White & 9 \\
\hline Silver & $10 \%$ Tolerance \\
\hline Gold & $5 \%$ Tolerance \\
\hline
\end{tabular}

$1^{\text {st }}$ Band $-1^{\text {st }}$ Digit

$2^{\text {nd }}$ Band $-2^{\text {nd }}$ Digit

$3^{\text {rd }}$ Band - Number of Zeros

$4^{\text {th }}$ Band - Tolerance 


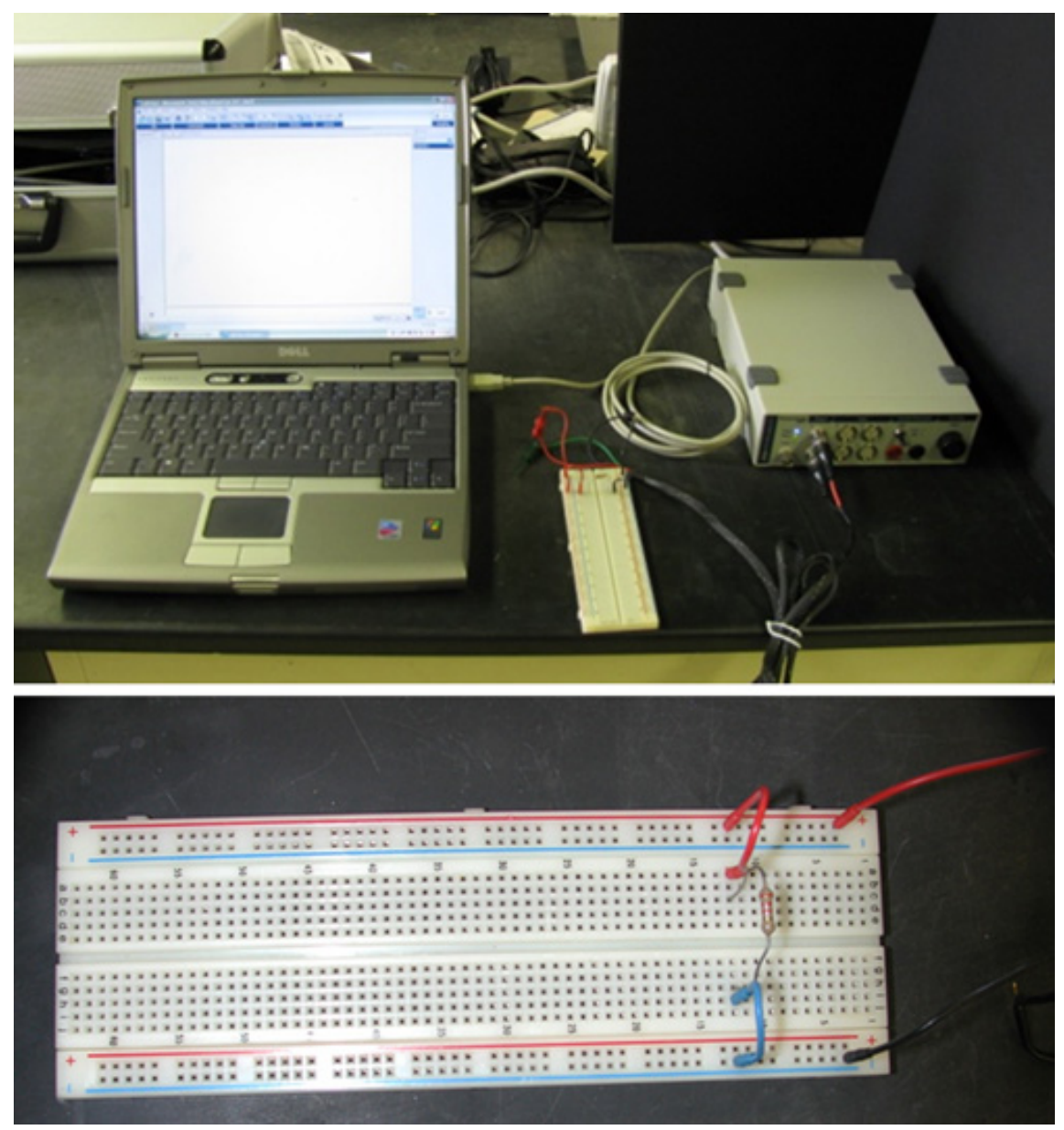

Figure 1: System Setup. Computer, bread board and power lab with output stimulator.

The PowerLab system (PowerLab interface from AD Instruments, Australia) will serve as the voltage source in this experiment. Attach the PowerLab's USB cable to the computer, turn on the PowerLab, and open the LabChart program from the desktop. Select "New File." A window will appear with multiple recording channels. Select "Setup" at the top and click on "Channel Settings." In the bottom left corner of the window, decrease the Number of Channels to 1; on Channel 1, change the Range to 5 V. Connect the Stimulator cable with the two mini-hook leads to the Output portals on the PowerLab: Attach the red connector cable to the positive Output portal and the black connector cable to the negative Output portal. Next, it is necessary to change the power output, frequency, and pulse duration of the PowerLab. In order to do this, select "Setup," and then "Stimulator Panel." Long pulses of $1.5 \mathrm{~V}$ are required for the first portion of the experiment, so adjust the amplitude to $0.75 \mathrm{~V}$; this will give a range of $1.5 \mathrm{~V}$ (the PowerLab will emit a voltage fluctuating between positive and negative $0.75 \mathrm{~V})$. Set the frequency $(0.5 \mathrm{~Hz})$ and pulse duration (1 s). 


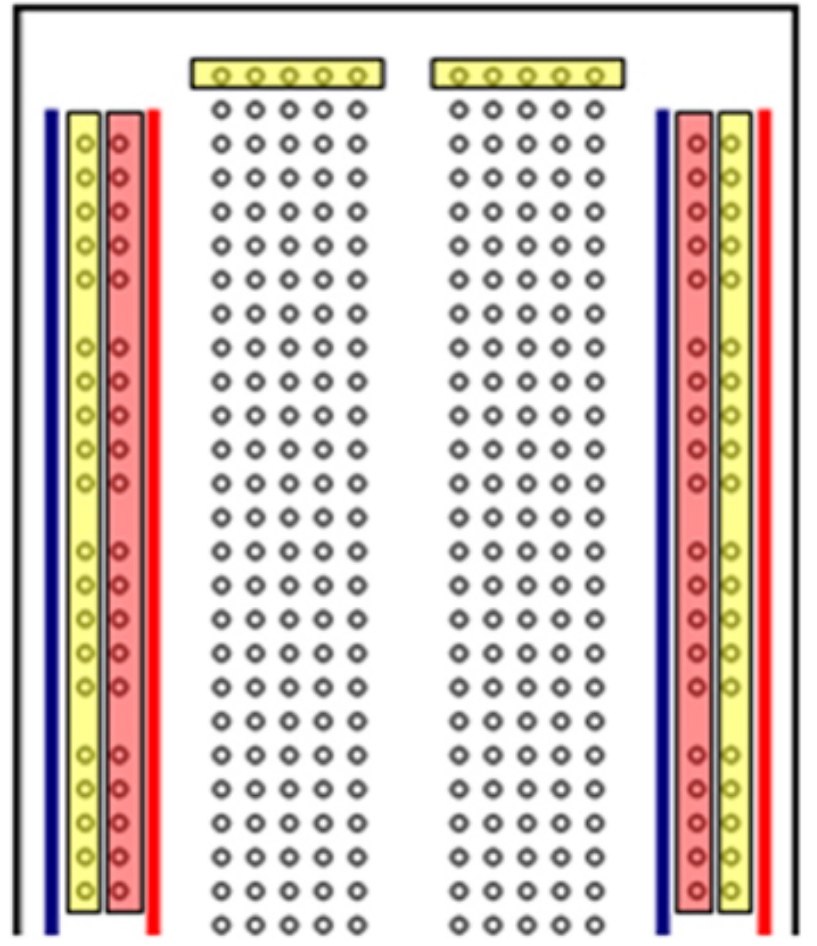

Figure 2: Breadboard schematics. The color strips along the sides are all connected as one unit for a given color. The 2 horizontal columns in the center are connected on each half, for a given row, but do not cross over the midline or to the side strips. Each row in the middle is independent of the next row.

When using a breadboard there are two columns on either side used for the input and output leads. Positive is generally connected to the right positive column in which all of the spaces in the column are connected. Negative input is connected to the opposite side of the breadboard where, again, all of the spaces in a column are connected. In the middle, between the two sets of columns, are multiple rows of five cells, each of which is independent of the others. All of the cells in a row of five are connected but isolated from other rows. Refer to Figure 2. The highlighted regions show what parts of the breadboard are interconnected. A useful video on breadboard use can be found online at http:// www.youtube.com/watch?v=oiqNaSPTI7w\&feature=related

\section{Exercise 1}

Use Ohm's Law to calculate the CURRENT flowing through the following circuit:

\section{NETWORK 1}

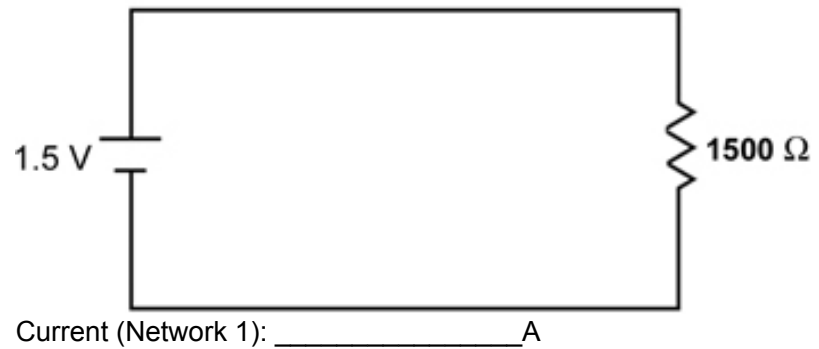

Exercise 2

Measure the voltage across the resistor, the resistance of the resistor, and the current flowing through the above circuit (Network 1). 


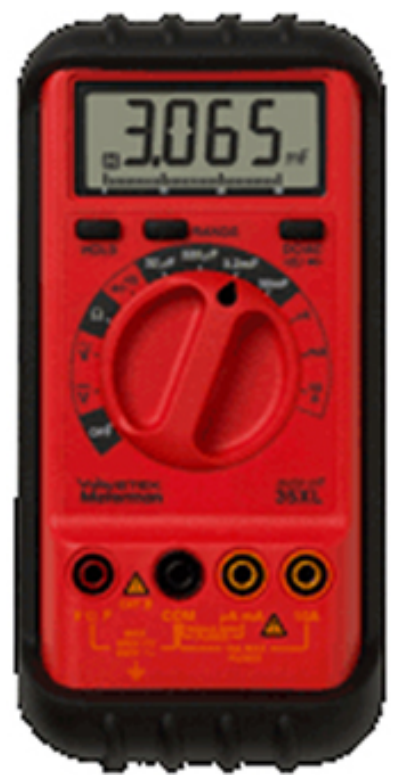

Figure 3: Wavetek Meterman voltmeter.

Recreate Network 1 on the breadboard using a $1500 \Omega$ resistor; the PowerLab serves as the voltage source (1.5 V). Measurements of voltage, current, and resistance are made with the Volt meter. In order for the Volt meter to gather accurate data, both the red and black probes must be in contact with bare wire in circuit. Also, the dial should be adjusted to DC voltage in a range appropriate for the scale of any individual experimental setup. For the voltage measurement, touch both probes of the Volt meter across the two ends of the resistor. When measuring the resistance of the resistor (not the voltage drop), DISCONNECT the voltage source and, again, touch both probes across the resistor. To measure current, the Volt meter must be in SERIES in the circuit; take note that the red and black probes must be oriented properly in reference to the current flow.

Voltage (Network 1):

Resistance (Network 1): $\mathrm{V}$

Current (Network 1): $\Omega$

Now with the Power Lab measure the voltage across Network 2 (same as Network 1, but on a bread board), just as you did with the volt meter. Disconnect the voltmeter and connect the leads for the voltage measuring probe with the red and black clips. Next proceed to use the cursor to click on the "Channel 1" (right hand side of screen). Click and go to "input amplifier". Then in the box click on the "differential" button and set range to $5 \mathrm{~V}$. Click "OK" to save changes.

Click on the "Start" (lower right hand corner) to collect data. One can stop at any time to measure the voltage deflections. To measure the voltage collected click "Stop" and use the "M" cursor (lower left corner) and move it to the baseline by dragging it over. Then use the free cursor to the top of the voltage trace to measure the amplitude. Read the change in voltage $(\Delta \mathrm{V})$ and record the data on paper. You can confirm the results with the stand alone voltmeter.

\section{4) Resistance and Conductance in Series}

Principle of the Voltage Divider: The voltage drop across the total of all the resistances in series is equal to the voltage of the source. The voltage across each individual resistor depends on the fraction of the total resistance that resistor represents. Hence:

$V_{1}=V_{(\text {source })} \frac{R_{1}}{R_{1}+R_{2}}$

A pair of resistors will divide a voltage in the ratio of their individual resistances.

Exercise 3

Calculate the voltage drop across each resistor in Network 3. Check your calculations by measuring the actual voltage with your volt meter and power lab software, with the $1.5 \mathrm{~V}$ source connected. 


\section{NETWORK 3}

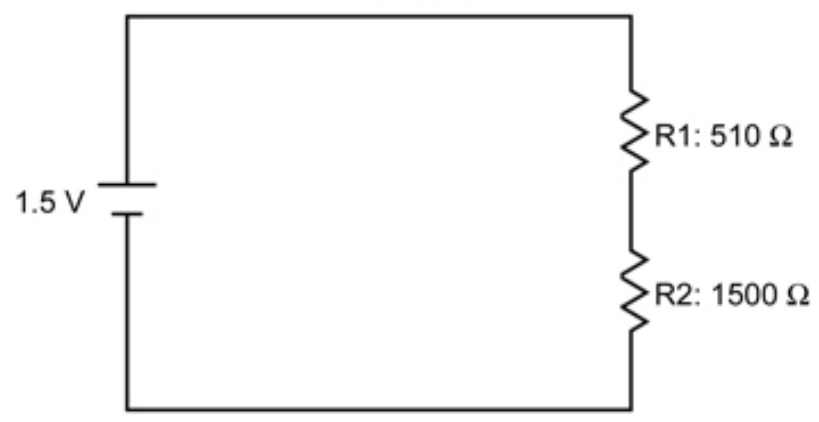

NETWORK 3. Use the two resistors indicated above.

\begin{tabular}{|c|c|}
\hline Calculated: & Measured: \\
\hline Voltage $\left(\right.$ Across $\left.\mathrm{R}_{1}\right)$ : $\quad \mathrm{V}$ & Voltage $\left(\right.$ Across $\left.\mathrm{R}_{1}\right)$ : \\
\hline Voltage $\left(\right.$ Across $\left.\mathrm{R}_{2}\right)$ : _ $\quad \mathrm{V}$ & Voltage (Across $R_{2}$ ): \\
\hline
\end{tabular}

\section{5) Sample Biological Application:}

The differing electrical properties of cell interior and external solution of an active nerve cell provide an example of a voltage divider. This biological circuit can be explored through recordings taken with external electrodes. The active region of the nerve cell, where ions enter, acts as a voltage source; current flows from this region along the interior of the cell, and returns through the external solution. Thus, between two points of the membrane that differ in potential there is a current flow, of necessity the same inside the fiber as out; since the resistance of the inside is larger than that of the outside, the greatest voltage drop occurs here.

Consider the system and its equivalent circuit (Figure 4: A. and B.):

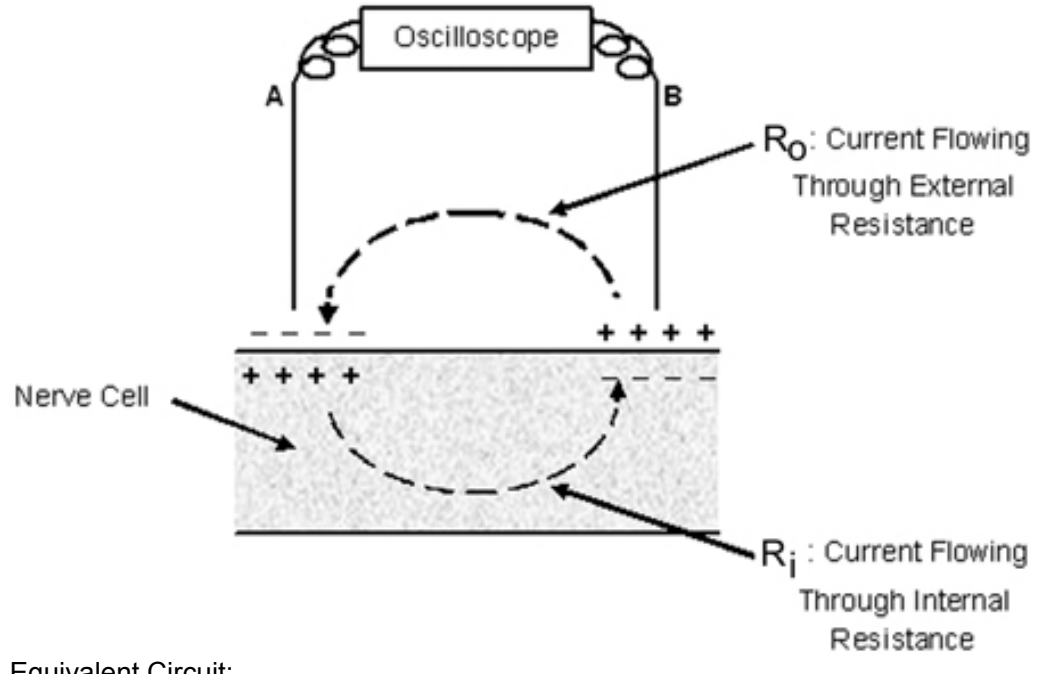

Equivalent Circuit:

Resistance

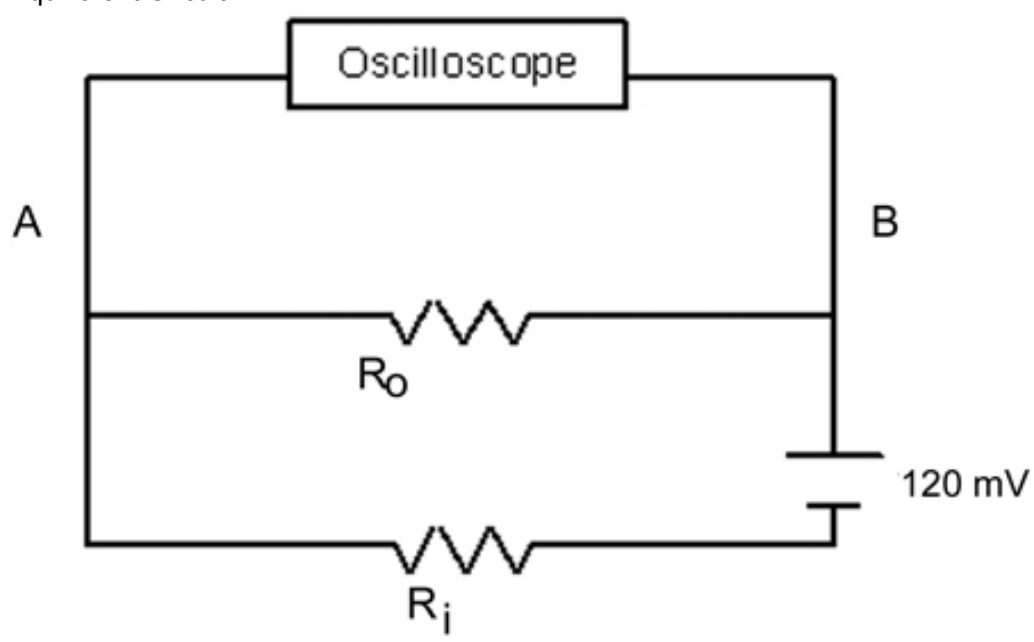


Figure 4: A (top). Electrical flow of current across a biological cell membrane. B (bottom). Equivalent electrical circuit. The voltage source $(120 \mathrm{mV})$ is the transmembrane voltage generated by the nerve cell's action potential.

Note: $R_{0}$ and $R_{i}$ are actually in series (not in parallel) due to the position of the power source. An equivalent drawing of the circuit is as follows:

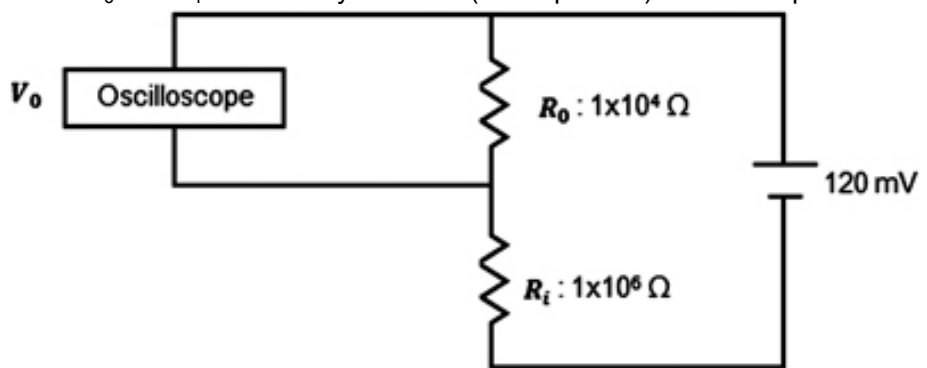

Figure 5: Alternate equivalent electrical circuit for Figure 4A.

Exercise 4.

Given that:

$\mathrm{V}_{\mathrm{m}}$, potential across membrane $=120 \mathrm{mV}$

$\mathrm{R}_{\mathrm{i}}$, resistance of internal fluid $=10^{6} \Omega$

$R_{0}$, resistance of external fluid $=10^{4} \Omega$

Calculate $V_{0}$, the potential between electrodes $A$ and $B$.

$\mathrm{V}_{0}$ : $\mathrm{v}$

Exercise 5.

Using Network 4 (see below), measure the potentials across each resistor $\left(R_{0}\right.$ and $\left.R_{i}\right)$.

NETWORK 4. Connect $4.7 \mathrm{~K} \Omega$ and $10 \mathrm{~K} \Omega$ resistors in series with the $1.5 \mathrm{~V}$ source:

\section{NETWORK 4}

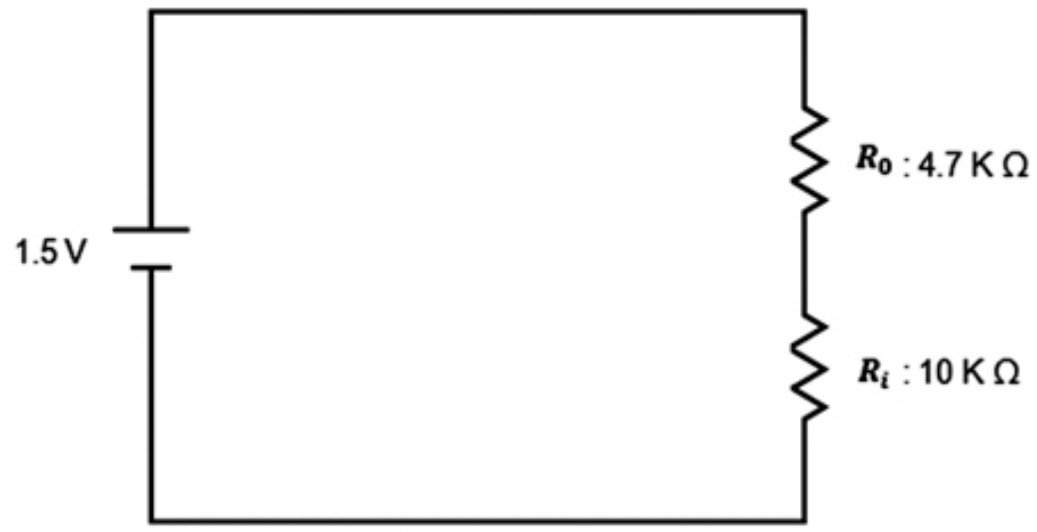

Measure voltages with your Volt meter.

Voltage (Across $\mathrm{R}_{0}$ ):

Voltage (Across $\mathrm{R}_{\mathrm{i}}$ ):

NETWORK 5 : Same as Network 4, except $R_{0}=1 \mathrm{~K} \Omega$

Note that in Network 5, the external resistance is much lower (analogous to having a lot of fluid or inactive tissue shunting the electrodes), hence the importance of recording from nerves in air or under insulating oil, to increase the external resistance.

Voltage $\left(\right.$ Across $\left.\mathrm{R}_{0}\right)$ :

Voltage (Across $\mathrm{R}_{\mathrm{i}}$ ): V

\section{6) Resistance and Conductance in Series and Parallel}

Sample Biological Application:

A good example of resistances in series and in parallel is the electrical configuration of a chemically transmitting synapse. Electrical transmission is not possible across most synapses because of the shunting effect of the synaptic gap. Note, however, that this shunt is probably necessary to allow the development of a large potential across the pre-synaptic membrane with attendant transmitter release. 


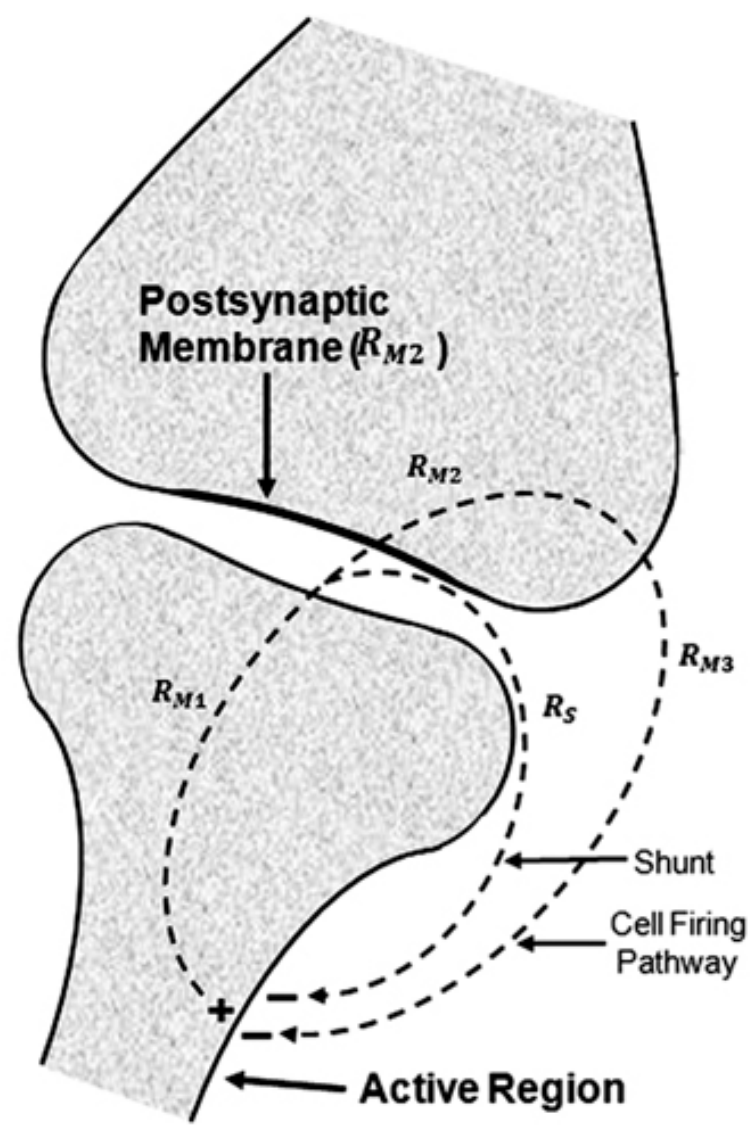

Figure 6: Model of electrochemical flow through a synapse.

NETWORK 6

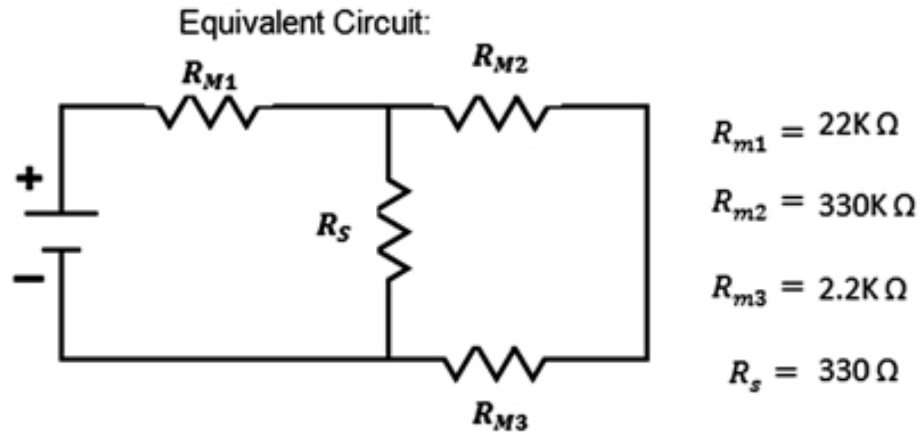

Note that in living tissue, significant electrical resistances are formed at membranes and in restricted extracellular spaces. Relatively large fluid spaces, such as cell cytoplasm or the bathing solutions, do not represent significant resistances.

\section{Exercise 6.}

Network 6 (see above, and Circuit Board) is an analog of a chemical transmitting synapse. In electrically transmitting synapses, the synaptic gap (shunt resistance $R_{\mathrm{s}}$ ) is eliminated and the current flows directly from presynaptic to postsynaptic cell through $R_{m 1}$ and $R_{m 2}$, then across $R_{m 3}$. The attenuation of the network can easily be measured with various values of resistor, illustrating changes in different synapses. Connect your PowerLab with 1.5V ( i.e., 0.75 Volt setting).

1. Measure the potential difference (PD) across each resistor in the network as provided, using the multimeter. Note the values of the resistors. Make a special note of the PD across $R_{m 3}$. (the membrane of the postsynaptic cell) Voltage: $\mathrm{V}$

2. Replace the $330 \Omega$ resistor at $R_{\mathrm{s}}$ with a $330 \mathrm{~K} \Omega$ resistor. Measure the $\mathrm{PD}$ at $R_{m 3}$

3. Now, keeping $R_{\mathrm{s}}$ at $330 \mathrm{~K} \Omega$, replace the $330 \mathrm{~K} \Omega$ resistor at $R_{m 2}$ with a $33 \mathrm{~K} \Omega$ resistor. Measure the PD across $R_{m 3}$.. Voltage:

4. Now keeping $R_{\mathrm{s}}$ at $330 \mathrm{~K} \Omega$ and $R_{m 2}$ at $33 \mathrm{~K} \Omega$, replace the $22 \mathrm{~K} \Omega$ resistor at $R_{m 1}$ with a $220 \Omega$ resistor. Measure the PD across $R_{m 3}$. Voltage: V

Exercise 7 
An example of series-parallel resistive network occurs when considering the cable properties of the nerve or muscle fiber. The LENGTH CONSTANT of a fiber is that distance over which a PD across the membrane declines to $37 \%$ of its original value. The attenuation is due to the internal and external resistances of the fluid on either side of the membrane being shunted by the (relatively high) transverse membrane resistance. The value of the resistance limits the transfer of a signal along a nerve cell's axon. The length constant is $1 / \mathrm{e}$ as measured for an exponential decay. $d E / d T=-E / R C$; where $E$ is the voltage difference, $R$ is resistance and $C$ is capacitance. Or in another form $E=E_{o} e x p(-t / R C)$; where $E_{o}$ is the starting voltage and $t$ is time in seconds. $R C$ is also referred to as tau $(T)$, the time constant.

Assuming the resistance of the external fluid to be negligible in comparison to the intracellular and membrane resistances, the passive resistive properties of the membrane can be represented by the following circuit:

\section{NETWORK 7}

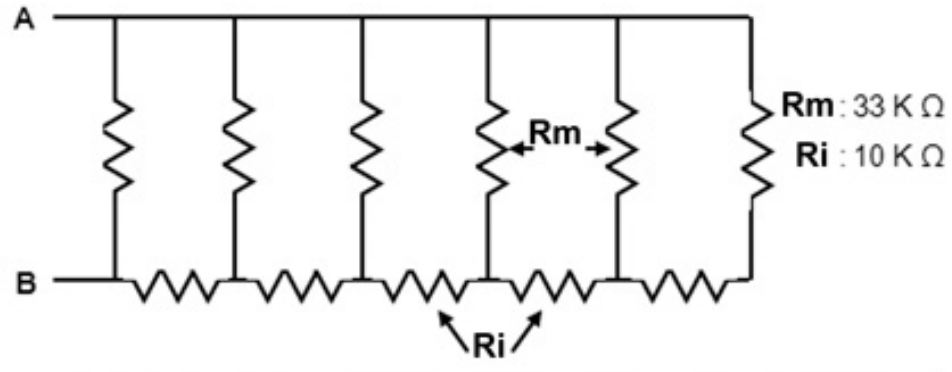

\begin{tabular}{|llllll|}
\hline 1 & 2 & 3 & 4 & 5 & 6 \\
& Unit Distance & for Length & Constant Calculations & \\
\hline
\end{tabular}
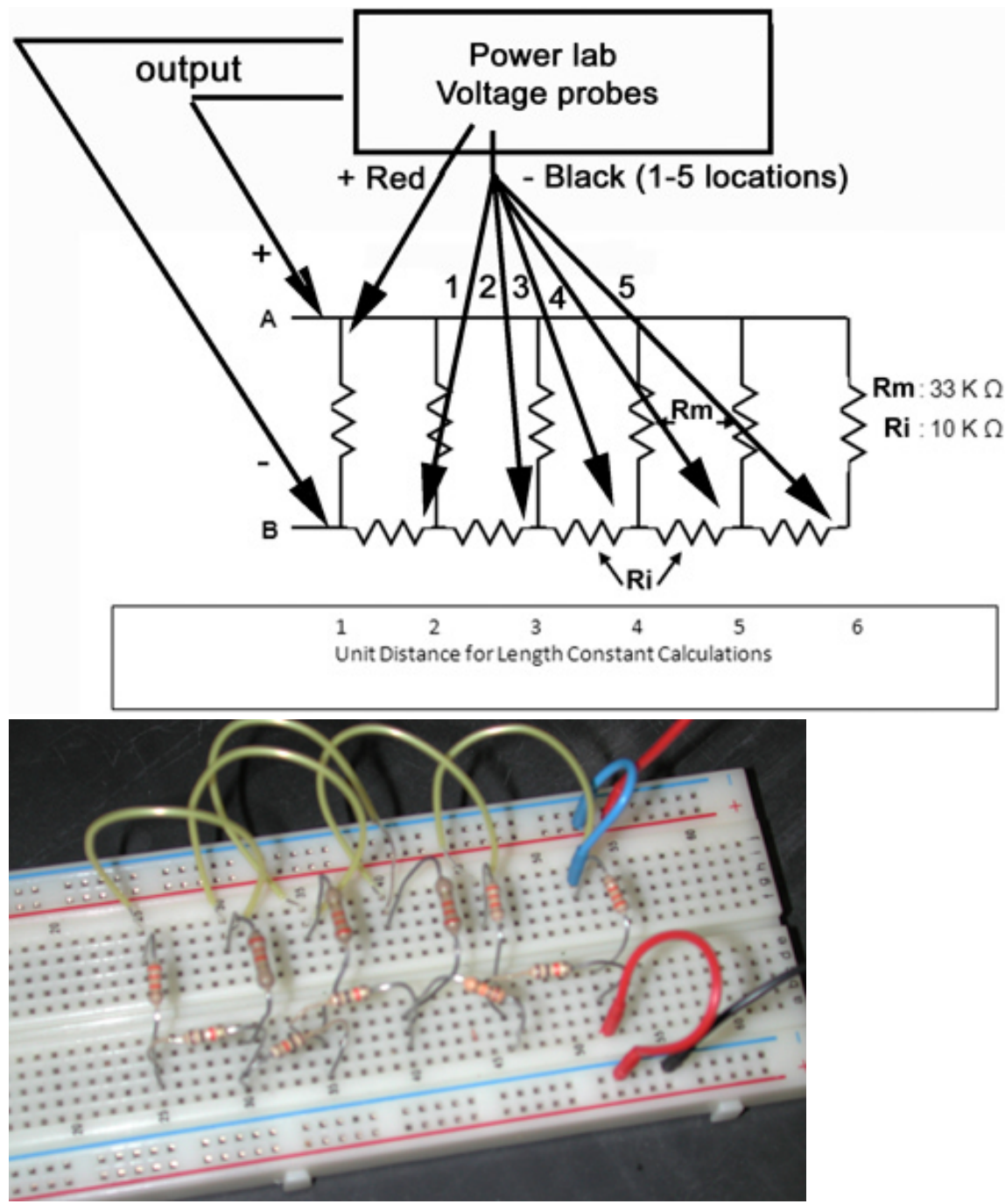

Figure 7: Translation of Network 7 onto breadboard setup. 
Apply a voltage at one end of Network 7 between A and B. Use a stimulator, selecting a pulse of $1 \mathrm{~s}$ duration, $1 \mathrm{~V}$ amplitude (set to $0.5 \mathrm{~V}$ ) and frequency at $0.75 \mathrm{~Hz}$. Connect the volt meter across each "membrane" resistor in turn, and note the PD across that resistor. Leave one end of the voltmeter probe stationary at (A-lead, Red) while the other lead is moved successively along the 1-5 locations. Plot each value on a graph of PD against "distance" from the source, the membrane resistors being unit distance apart. From your graph determine the length constant of this

\section{Potential Difference vs. Distance}

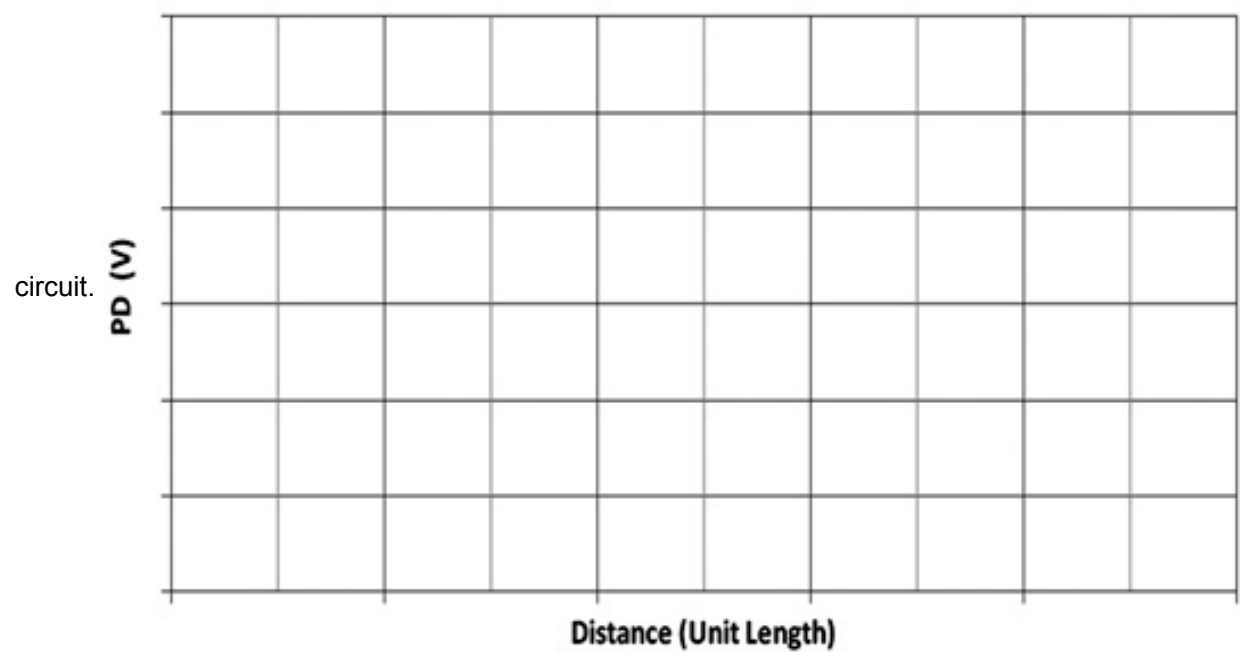

Graph of Network 7 Length Constant:

The effect of an increase in transverse membrane resistance, as would occur with a myelin sheath, or a decrease in longitudinal internal resistance of the fiber associated with an increase in fiber diameter, is a change in length constant. The value of the resistors is not those actually found in a nerve axon, but is approximately proportional. Likewise, the "unit distance" on the model is substantially scaled up from a real nerve cell.

\section{7) Capacitance}

Capacitors store charge. If a voltage is applied to a capacitor through a resistance, current flows into the capacitor, and then the PD (potential difference) across the capacitor rises exponentially with time toward the PD of the source; the time course of this voltage change is dependent on the applied voltage, the capacitance, and the series resistance. A similar but inverted time course is seen when a capacitor discharges.

\section{NETWORK 8}

\section{A O-}

\section{$\mathrm{B} \mathrm{O}$}

Connect the input of this simple Resistance-Capacitance $(R C)$ network to your stimulator, at $A$ and $B$. Use $1 \mathrm{~V}$ (set to $0.5 \mathrm{~V}$ ) pulses at duration of $1 \mathrm{~s}$ and frequency of $0.75 \mathrm{~Hz}$. Also one must set the acquisition to $20 \mathrm{~K} / \mathrm{sec}$ (right hand side of panel) Connect your input clamps from the PowerLab across the capacitor. Collect a few seconds and then stop. Use the Zoom window to expand the rise time on one of the square pulses. The zoom in might have to be repeated a few times to spread out the trace. Measure the rise time from baseline to the top of the voltage trace just as it levels off. Observe the time course of potential change, and the distortion of the square pulses. Observe the effect of changing the $10 \mu \mathrm{F}$ capacitor for the one of $0.1 \mu \mathrm{F}$. By connecting your power lab volt probe across the resistor, you can observe the time course of current flow through the circuit. Collect only a few pulses and then stop to measure. Use the "M" cursor to move to the base line prior to the rise and the free cursor to where the voltage begins to levels off .

The last example involves both resistive and capacitative components. The passive cable properties of nerve and muscle fibers are determined by similar elements, arising from cell membrane properties. Not only is there a transverse membrane resistance present in the cell, but also a 
membrane capacitance, due to the electrical properties of the cell membrane. The effect of this is to affect the time course of the development of electrotonically spread potentials.

Example:

\section{NETWORK 9}

A

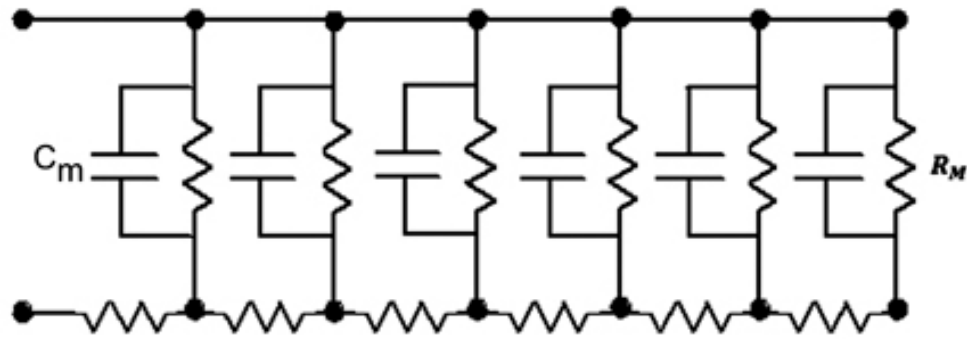

B

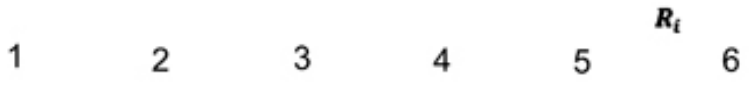

Unit Distance for length Constant Calculations

This network can be constructed from Network 7 by attaching the required capacitors in parallel to the membrane resistors (Rm).

\section{Exercise 8.}

Attach to Network 7 the $10 \mu \mathrm{F}$ capacitors. Using $1 \mathrm{~s}, 1 \mathrm{~V}(0.5 \mathrm{~V}$ setting $)$ and $0.75 \mathrm{~Hz}$ pulses from the stimulator, which should be connected at A and $\mathrm{B}$, observe the potential changes across each membrane resistor. Assuming the "threshold" voltage (the voltage at which an action potential is generated in a nerve cell) to be $0.2 \mathrm{~V}$, measure the time taken for the potential to reach threshold at each membrane resistor. Threshold here means the potential value at which an action potential can be generated in a living cell. Plot your results as time against 'distance.'

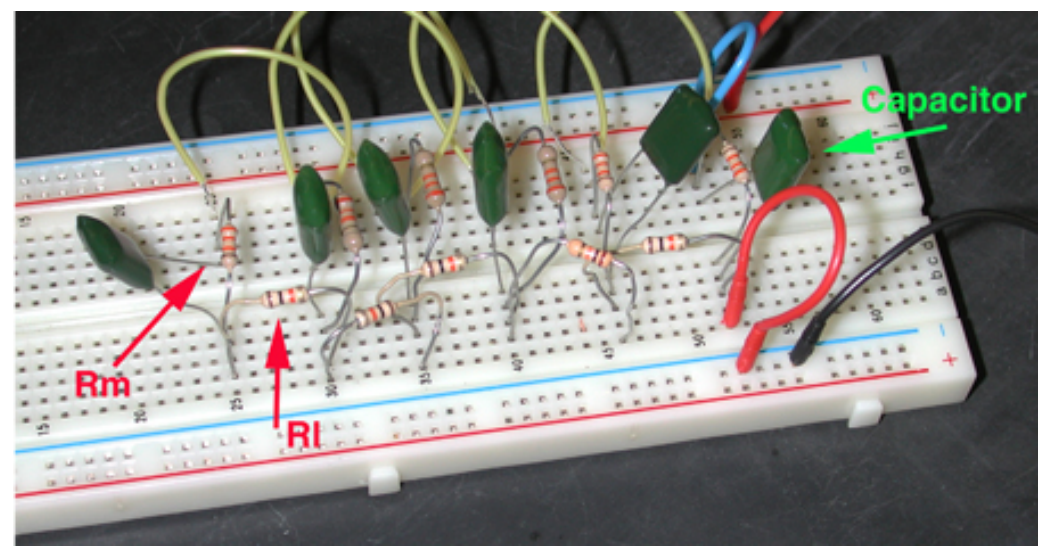

Figure 8: Translation of Network 9 onto breadboard setup.

\section{Time vs. Distance}

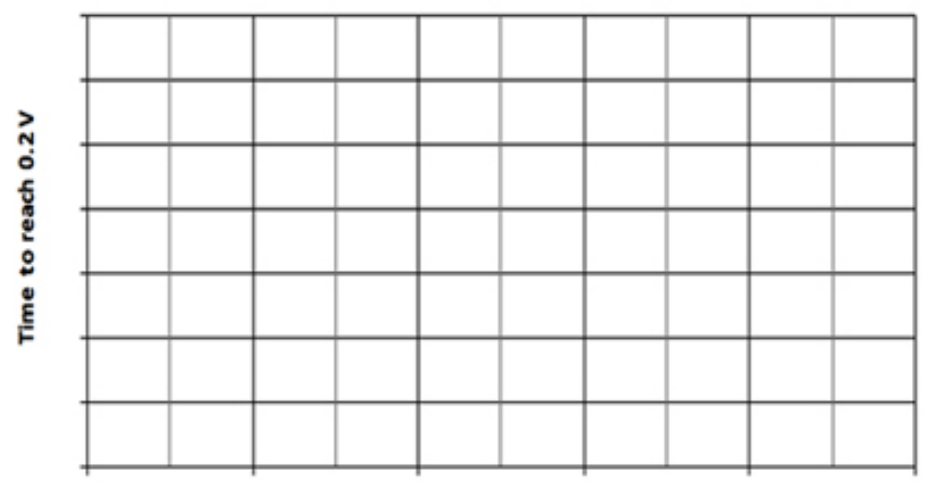

Graph for Figure 8.

Distance (Unit Length) 
Simulate the effect of a decrease in membrane capacitance produced by an increase in thickness of the myelin sheath by substituting to the smaller $0.1 \mu \mathrm{F}$ capacitors. Note the change in time to reach threshold at a selected resistor. Superimpose the data on the same graph with the larger capacitors in place.

In terms of a muscle membrane one can think of an increased capacitance due to increase in surface area of membrane by the associated Ttubules which results in a larger membrane capacitance as compared to a neuron. Smaller capacitors can be used to illustrate the decreased capacitance due to addition of myelin to an unmyelinated nerve axon. The main point here to learn is that the electrical capacitance is proportional to surface area, and inversely related to thickness of the dielectric layer (myelin) which separates the "plates" or storing surfaces of the capacitor.

The properties presented on a model circuit are observed in live preparations. However, cells vary in their properties such as leakage across the membrane and axial resistance. Some cells do not generate action potentials. However, an action potential is produced when a signal is needed to be transmitted over a few millimeters because passive electrical properties of cells attenuate signals over a few millimeters. In the next series of experiments the recording of action potentials is illustrated.

\section{8) Questions for the students}

1. What are some normal values of length constant for neurons and muscle cells?

2. What if a membrane had a low membrane resistance: would the time constant (time to reach $63 \%$ of final value) change? If so, how would it change with a lower membrane resistance? What is the generalized mathematical formula for time constant (tau)?

3. Why is it that larger axons, such as the squid giant axon, can conduct electrical signals faster than smaller axons in the same squid preparation?

\section{CONDUCTION PROPERTIES OF NERVE CELLS}

\section{1) From a bread board to living cells}

The passive properties demonstrated in the circuit board exercises are also measurable in living cells; however, these passive properties can be complicated to tease out when accompanied with action potentials in nerve cells. The active properties of the neuron are its ability to maintain a resting membrane potential, generate action potentials, and regenerate them along a length of membrane.

Electrical flow along a nerve cord can also be regenerative within individual neurons and from one neuron to the next. In the crayfish ventral nerve cord (VNC), some neurons communicate via septate (i.e. gap) junctions. As demonstrated in the earlier exercises with the bread board, altering the resistance can impede conduction of electrical signals. This is analogous to altering the flow of current through gap junctions within the ventral nerve cord.

The conduction velocity and other properties of the compound action potential in the VNC are examined in the next series of experiments. We will demonstrate how to obtain the VNC for experimentation and how to elicit and measure the responses using standard equipment designed for teaching student laboratories.

\section{2) The crayfish preparation}

The crayfish ventral nerve cord is best suited for this experiment. The nerve is easily dissected, and a variety of protocols that effect conduction can be used with this preparation. Before starting this experiment, become familiar with the crayfish anatomy terms below:

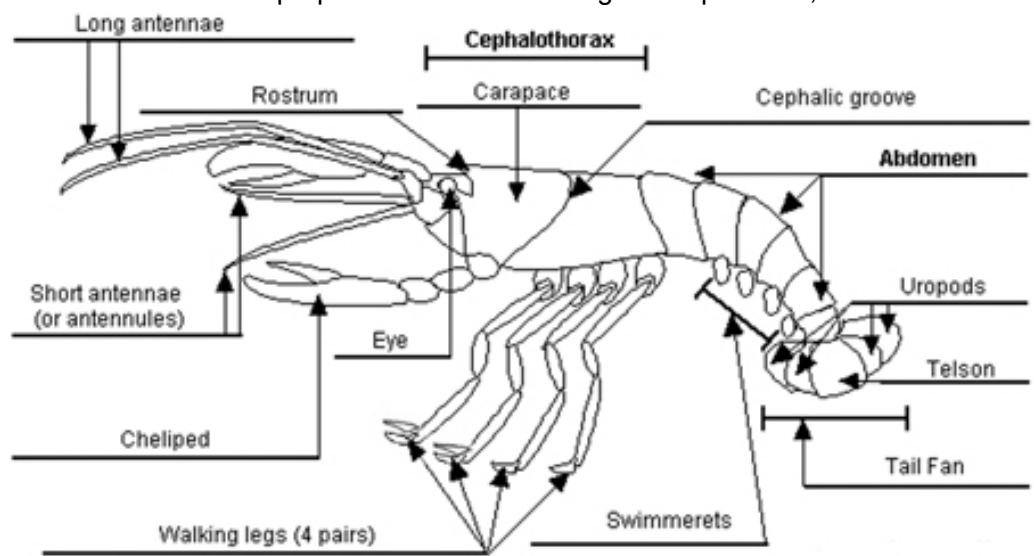

Figure 9. General crayfish anatomy diagram.

Modified from: http://www.enchantedlearning.com

\section{3) Background on conduction in a nerve}

The crayfish ventral nerve cord functions in transmitting signals that initiate escape responses such as the tail flip reaction. For this experiment, recordings will be taken from the entire intact nerve cord. The individual potentials from each neuron within the nerve cord sum together to give rise to the compound action potential, which provides the measurable electrical pulses shown by the recording software. Erlanger, Gasser, and Bishop (1924) are some of the pioneers who described compound action potentials. Characteristics of the refractory period, conduction velocity and the neural effects of the gap junction inhibitor 1-heptanol or temperature are shown. Learning the basic principles of nerve cell signals 
contributes to a better understanding of neurological function in general (Bennett, et al., 1991; Furshpan et al., 1959; Watanabe and Grundfest, 1961).

\section{4) Materials}

\begin{tabular}{|l|l|}
\hline Item & Quantity \\
\hline Large Crayfish (Procambarus clarkii) & 1 \\
\hline Precision Dissecting Scissors & 1 \\
\hline Forceps & 1 \\
\hline MLT016/X Nerve Chamber & 1 \\
\hline Laptop w/Scope software & 1 \\
\hline PowerLab & 1 \\
\hline PowerLab Amplifier & 1 \\
\hline Stimulator output cable for PowerLab & 1 \\
\hline USB PowerLab/Computer Connector & 1 \\
\hline Manipulator Stand with Suction Electrode & 1 \\
\hline Glass Pipette w/bulb & 1 \\
\hline Beaker of Crayfish Saline & 1 \\
\hline
\end{tabular}

\section{5) Methods}

\subsection{1) Dissection}

Ensure that the nerve dish, various instruments, and solutions are prepared before dissecting a crayfish.

1. Wrap crayfish in moist paper towel to hold or just hold crayfish in one hand with claws (chelipeds) between thumb and forefinger.

2. Using the other hand, make an incision with scissors through rostrum between eye sockets.

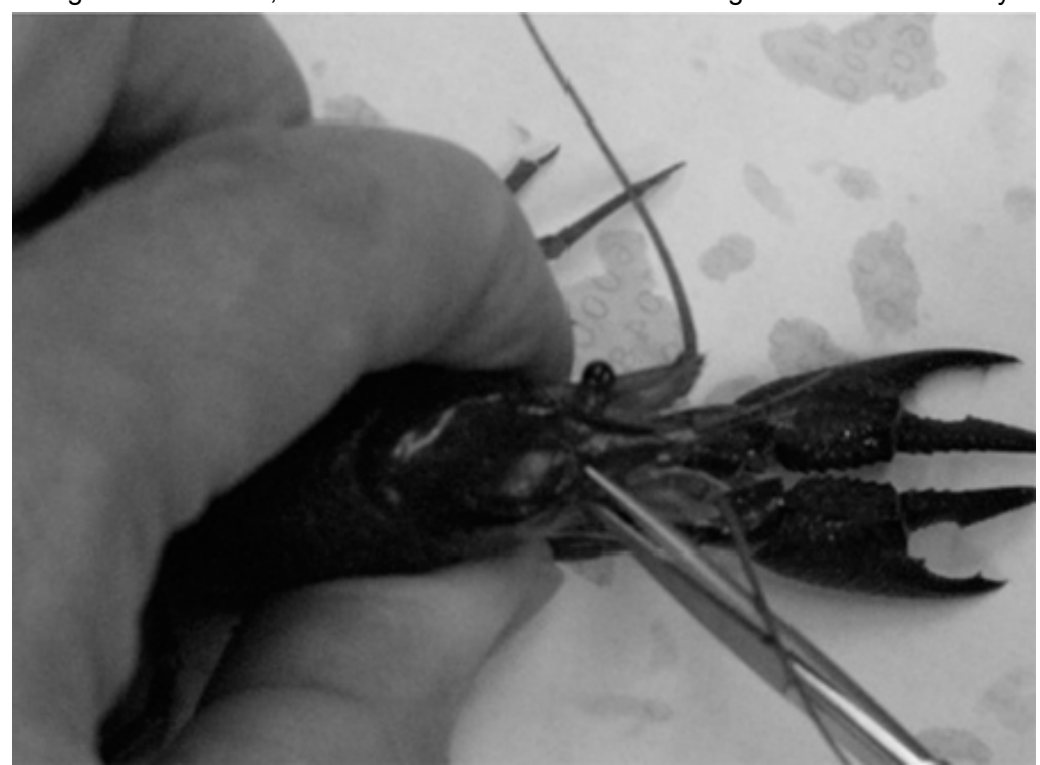

3. Cut downwards from the incision until the head is completely severed.

4. Make an incision at base of cephalothorax above the abdomen; remove the tail. (If needed, chelipeds and walking legs may be removed to facilitate this process.) 


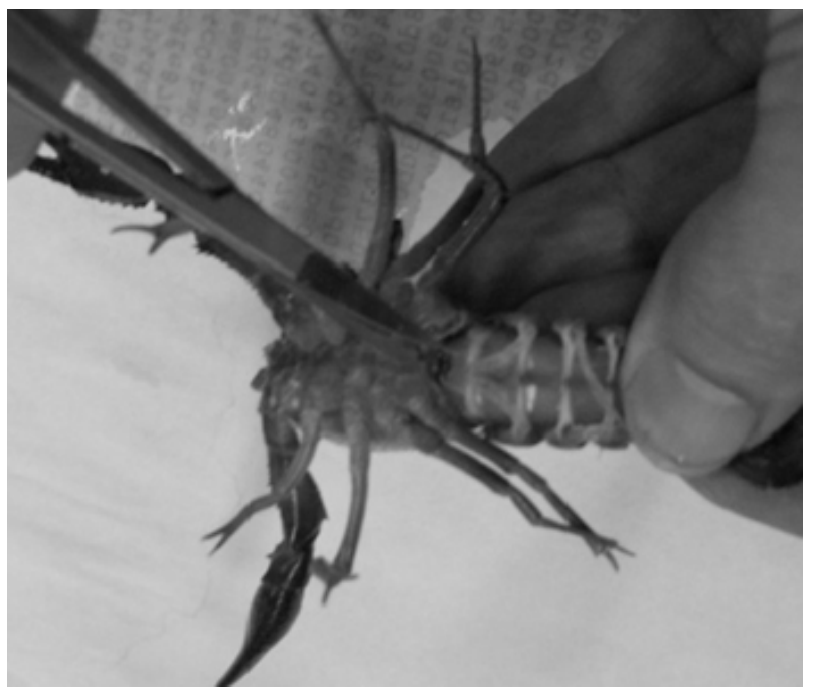

5. Remove the swimmerets.

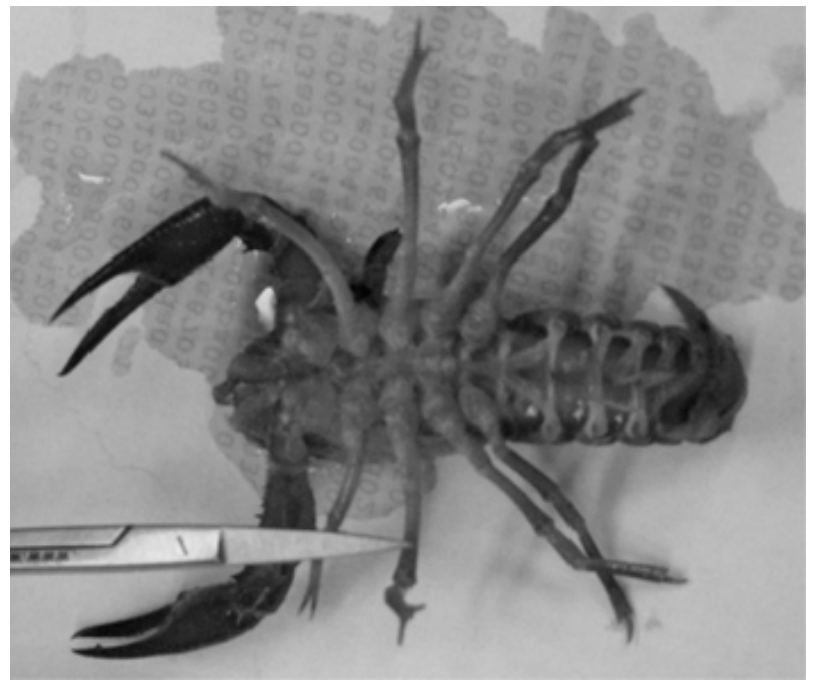

6. Make a cut along both sides of the ventral side of the abdomen. Be careful to not damage deeper tissue.

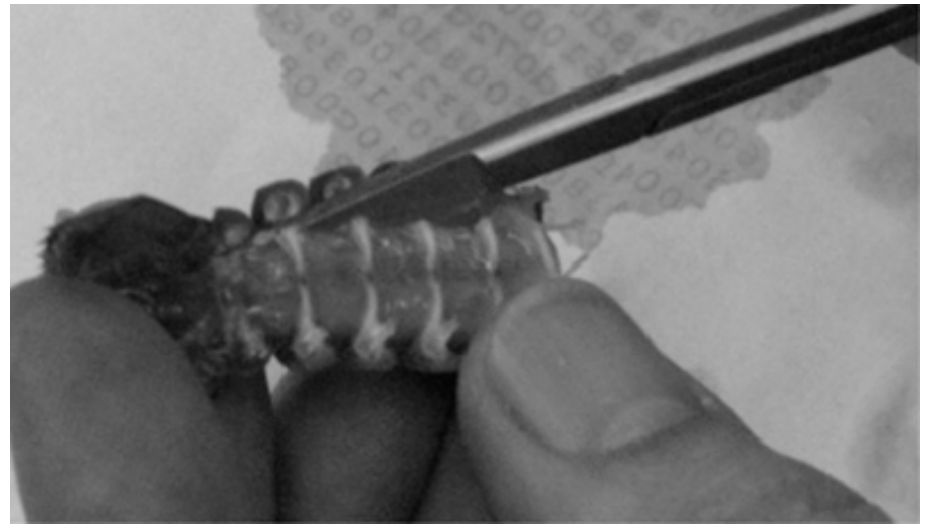

7. Peel back the freed section of exoskeleton to the base of the tail fan in order to expose the ventral nerve cord. 


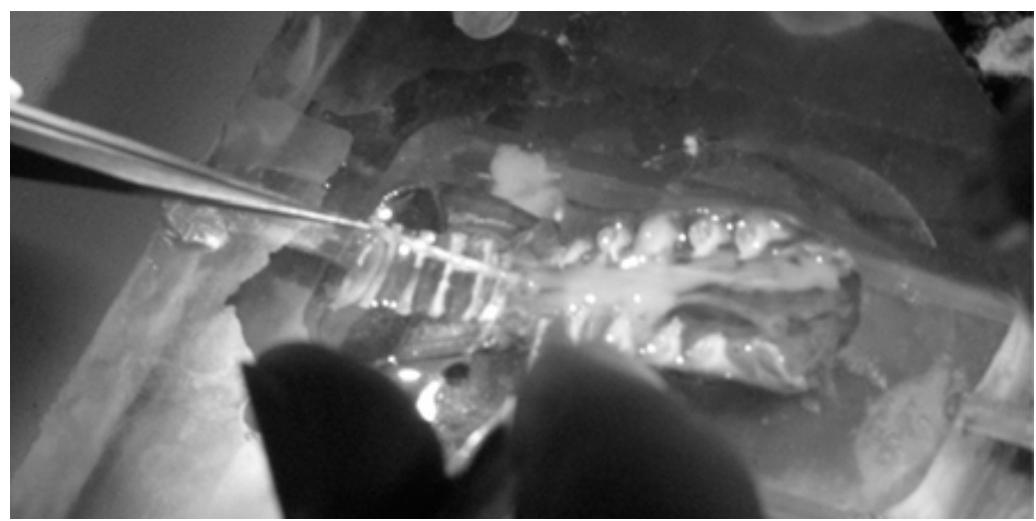

8. Using the edge of the forceps, carefully remove the ventral nerve cord by the tip. Be careful not to stretch or crush the nerve cord. (Any nodules observed on the nerve cord are crayfish ganglia.)

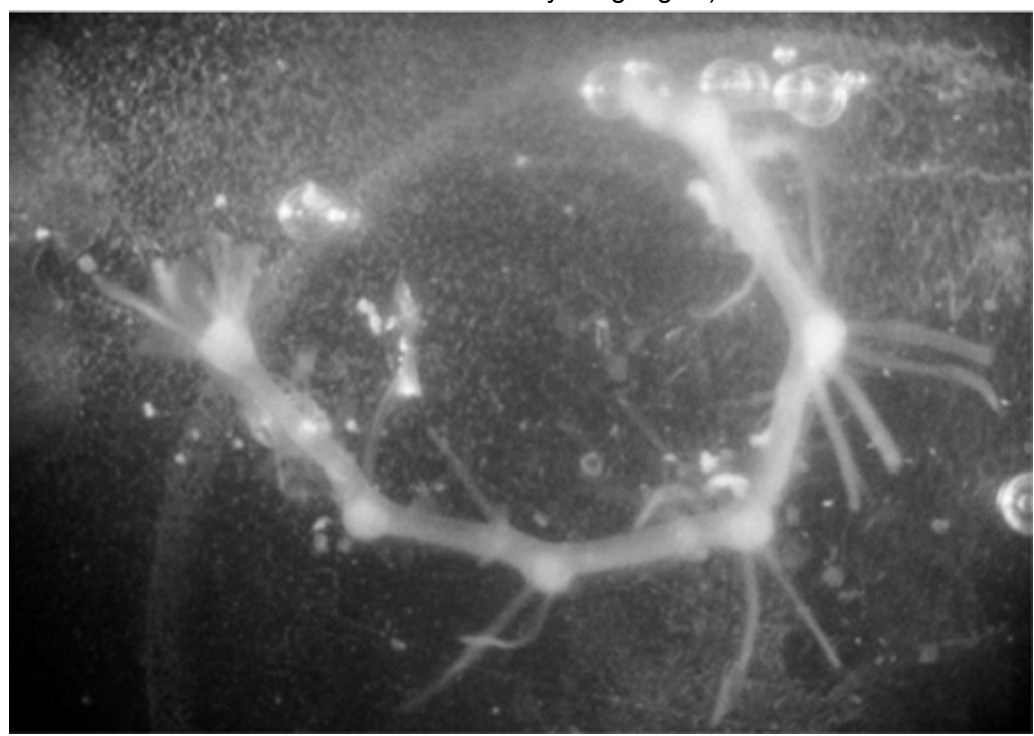

9. Lay out the nerve cord across the metal bars in the nerve chamber. It is imperative to keep the ventral nerve cord moist. To accomplish this, add enough crayfish saline solution to the nerve chamber to reach the level even with the metal crossbars. Next, pipette out enough of the crayfish saline solution so that the level of the liquid just makes contact with the bottoms of the bars.

Crayfish Saline (mM: $205 \mathrm{NaCl} ; 5.3 \mathrm{KCl} ; 13.5 \mathrm{CaCl}_{2} .2 \mathrm{H}_{2} \mathrm{O} ; 2.45 \mathrm{MgCl}_{2} \cdot 6 \mathrm{H}_{2} \mathrm{O} ; 5 \mathrm{HEPES}$ adjusted to $\mathrm{pH} 7.4$ )

10. Once the nerve is in place, attach the stimulator cable with the two mini-hook leads to the nerve chamber. Attach the positive red lead to one of the external metal loops near the end of the nerve cord; attach the negative black lead to the nearest adjacent metal loop still in contact with the nerve cord. 


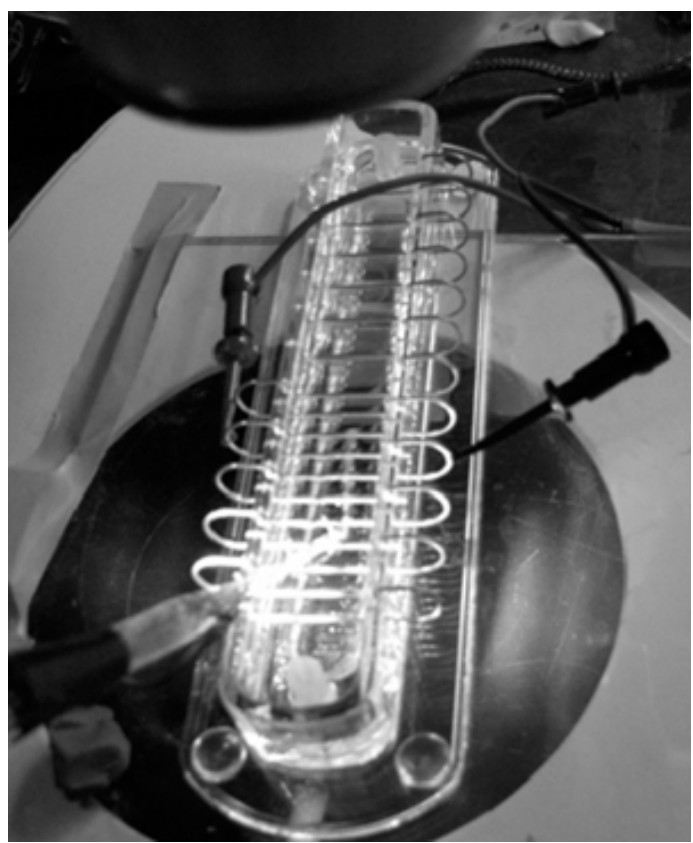

11. Position the suction electrode within the manipulator near the opposite end of the nerve.

12. Carefully suction the tip of the ventral nerve cord into the recording electrode.

\subsection{2) Recording}

Once the tip of the nerve cord is suctioned into the recording electrode, ensure that the electrode is attached to the probe and that the probe is sufficiently grounded. This can be accomplished by simply attaching the ground cable to the Faraday cage. The positive and negative suction electrode wires should also be attached to the corresponding locations on the probe. The probe should be attached to the differential amplifier which, in turn, is connected to the PowerLab.

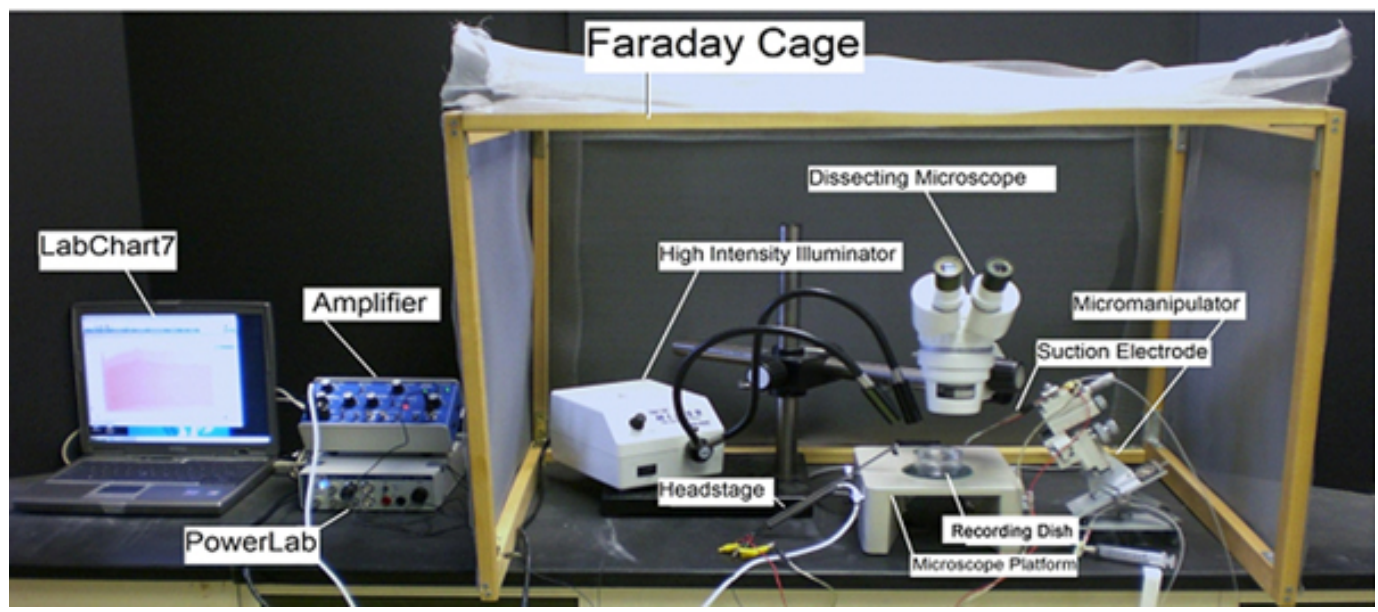

Connect the electrical wires from the suction electrode to the head stage. The wires should be connected with the red (positive) at the top left, green (ground) in the middle, black (negative at the bottom. This is indicated in Figure 10 for the head stage below. The ground wire can just be put in the saline bath. Thus, one wire from the suction electrode to the + and the other to the - input. It does not matter which wires from the suction electrode goes to the + or - inputs.

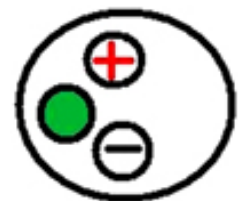

Figure 10: Inputs for the head stage

The settings for the amplifier are as follows:

\begin{tabular}{|l|l|}
\hline CONTROL & SETTING \\
\hline High Pass & DC \\
\hline
\end{tabular}




\begin{tabular}{|l|l|}
\hline Notch Filter & OFF \\
\hline Low Pass & $20 \mathrm{kHz}$ \\
\hline Capacity Comp. & Counterclockwise \\
\hline DC Offset Fine and Course knob & Counterclockwise \\
\hline DC Offset (+OFF) & OFF \\
\hline Gain knob & 50 \\
\hline Input (DIFF MONO GND) & DIFF \\
\hline MODE(STIM-GATE-REC) & GATE \\
\hline STEST & OFF \\
\hline
\end{tabular}

Connect the provided USB cable to the back of the PowerLab and to the computer outlet. Open the Scope software on the computer desktop.

On the top right of the screen, select "Channel 2" and turn off. Adjust screen so only Channel 1 is showing by sliding bar in middle of screen to the bottom. Next, click on the "Input Amplifier" for channel 1 on top right of the screen. Select the following:

\begin{tabular}{|l|l|}
\hline CONTROL & SETTING \\
\hline RANGE & $500 \mathrm{mV}$ \\
\hline AC & CHECKED \\
\hline LOW PASS & OFF \\
\hline SINGLE ENDED & CHECKED \\
\hline INVERT & CHECKED \\
\hline
\end{tabular}

Next, again under "Set up," click "Sampling." In the box entitled "Sweep" on the screen that appears, select the following:

\begin{tabular}{|l|l|}
\hline CONTROL & SETTING \\
\hline MODE & MULTIPLE \\
\hline SAMPLE & 100 SWEEPS \\
\hline SOURCE & USER \\
\hline DELAY & 0 SECONDS \\
\hline
\end{tabular}

Finally, under the "Set up" tab, select "Stimulator" . Click on box to turn off "Isolated Stim". Choose the following settings:

\begin{tabular}{|l|l|}
\hline CONTROL & SETTING \\
\hline MODE & MULTIPLE \\
\hline DELAY & $5 \mathrm{~ms}$ \\
\hline DURATION & $1 \mathrm{~ms}$ \\
\hline PULSE(S) & 2 \\
\hline INTERVAL & $15 \mathrm{~ms}$ \\
\hline RANGE & $5 \mathrm{~V}$ \\
\hline AMPLITUDE & 0.5 \\
\hline & \\
\hline & \\
\hline
\end{tabular}

See "Time Base" in right hand panel. Change "Sample" to 1024 and "Time" to 200 msec. 


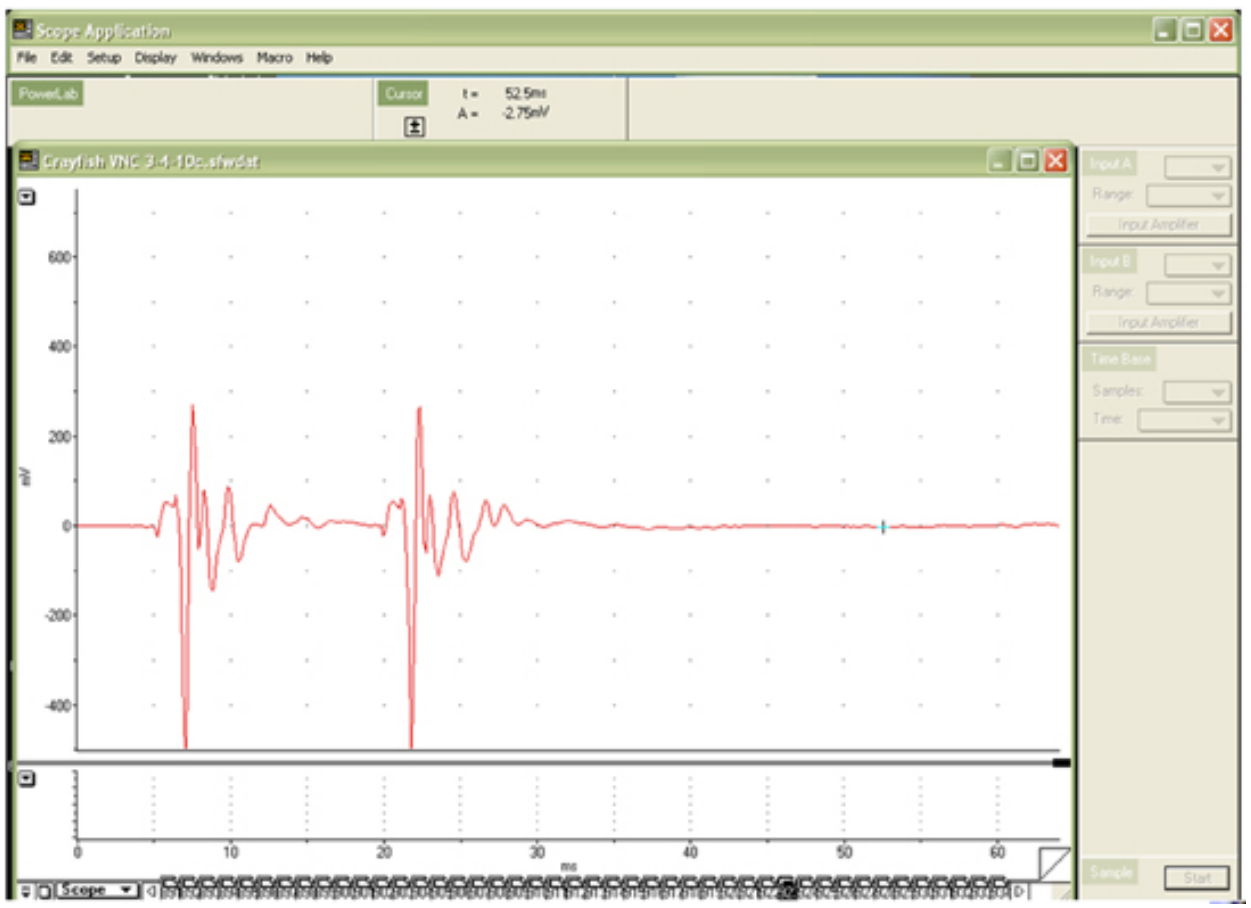

Figure 11: Two compound action potentials with $15 \mathrm{msec}$ interval between stimuli as recorded with the Scope software.

\section{Exercise 1.}

Select the "Start" button at the lower left of the screen. Given the above settings, a clearly defined compound action potential should appear on the Scope data collection box (Figure 11). Sketch the general shape of the action potential on the graph below (Figure 12):

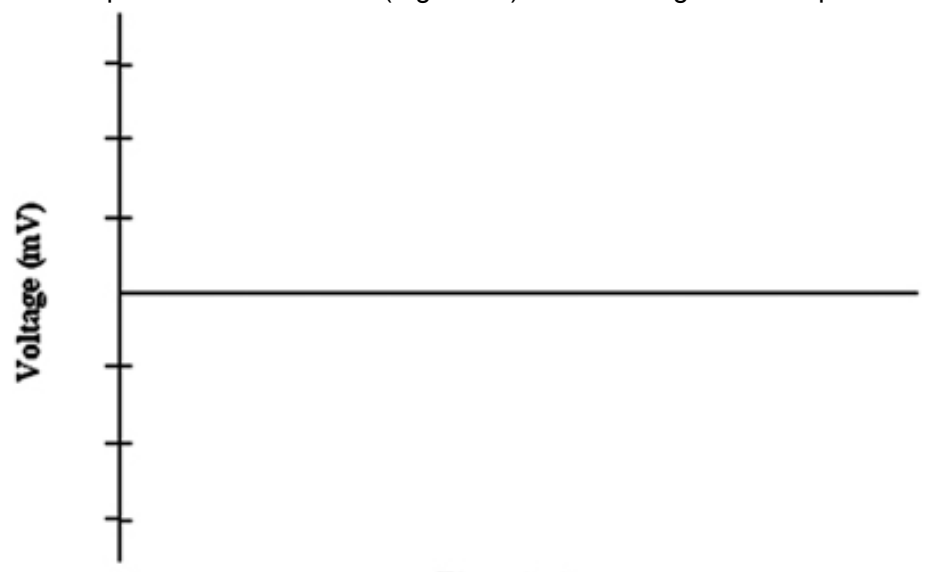

Time (ms)

Figure 12: Blank graph to sketch shape of compound action potential.

Slowly decrease the voltage using the tab at the top left of the screen. The action potential should become smaller in magnitude until its characteristic wave pattern likewise disappears. Decreasing the voltage reduces the number of neurons that are recruited to fire the compound action potential. The larger axons are likely the recruited at the lower voltages and are the faster conducting ones which would tend to appear first in the compound action potential. Incrementally increase the voltage to observe the opposite effect; as more neurons are recruited, the compound action potential wave grows larger. This exercise illustrates the fact that individual neurons have different thresholds for action potential production. When the compound action potential does not increase any further with increasing stimulating voltage, all the neurons have been recruited; two distinct peaks in the action potential wave on the computer screen should be visible.

Exercise 2.

After examining neuron recruitment and the resulting compound action potential, observe the effect of the refractory period. This can be done by giving two stimuli close together in time and varying the interstimulus interval, then observing at what interstimulus interval the compound action potential, elicited by the second stimulus, is reduced in amplitude compared to the first compound action potential. Change the delay between pulses using the tab in the top left corner of the screen. Keep shortening the delay until the second compound action potential cannot be elicited. The time between pulses at which a second action potential is no longer elicited is the absolute refractory period. The time between the end of the absolute refractory period to the time when a second stimulus generates an action potential of full amplitude is termed the relative refractory period. Give an estimate of the absolute and relative refractory periods in the spaces provided. 
Absolute Refractory Period:

Relative Refractory Period:

\subsection{3) Measuring conduction velocity}

With a millimeter ruler, measure the distance between the stimulating wire closest to the recording electrode, and the recording electrode. From the recorded trace, measure the time to the various peaks of events in the compound potential so that you can record conduction velocity of the various electrical signals. Convert the $\mathrm{mm}$ distance to meters and the $\mathrm{msec}$ time to $\mathrm{sec}$ for meters/second as units.

$1^{\text {st }}$ wave-form conduction velocity:

$2^{\text {nd }}$ wave-form conduction velocity:

$3^{\text {rd }}$ wave-form conduction velocity:

$4^{\text {th }}$ wave-form conduction velocity:

\subsection{4) Manipulation of the gap junctions and/or conduction velocity}

Next, investigate the properties of a neuronal inhibitor. The uncoupling effect of heptanol was first described in crayfish septate axons (i.e., gap junctions) by Johnston et al. (1980). This effect related to electrical communication was soon confirmed in various vertebrate and invertebrate systems (Bernardini et al., 1984; Meda et al., 1986). To understand the role of electrical communication in this ventral nerve cord, a saline with $(1 \mathrm{mM})$ 1-heptanol can be used to uncouple gap junctions. With a glass pipette, wash over the over the entire nerve cord a solution of heptanol. Observe the change that is recorded by the Scope recording software, and summarize it. (Note the change in the wave-form, and whether conduction velocity of the wave-forms has changed). Alternatively, instead of using 1-heptanol, ice-cold saline could be exchanged for the bathing saline and any change in the wave-form or conduction velocity of the wave-forms could be monitored.

\section{Discussion}

Our goal in the on-line video presentation and this paper is to demonstrate that the biophysical properties of cells can, in part, be modeled as electrical circuits. In addition, with live neural tissue that is relatively easily obtained, fundamental principles of conduction velocity, refractory periods and electrophysiological recording techniques are possible for undergraduate student laboratories with modest investment of equipment. The themes and fundamental paradigms presented can be readily modified for the requirements of different courses.

Maintenance of crayfish and their abundance makes them attractive models for student-driven experimentation. Crustacean ventral nerve cords are generally robust and retain physiological integrity in a minimal saline for hours, which is adequate for a 3 hour student laboratory.

Given that some of the large axons in the VNC of the crayfish are connected via gap junctions, additional experimentation on their contribution can be conducted, and different properties than found in the standard frog sciatic nerve preparation can be demonstrated. The sciatic nerve is a classic model for addressing compound action potentials and conduction properties. It might even be an interesting comparative experiment for students to compare conduction properties, axon recruitment, and refractory periods between these two preparations.

\section{Disclosures}

No conflicts of interest declared.

\section{Acknowledgements}

These experiments were modified from a laboratory manual that has been used in a course, orchestrated by Dr. H.L. Atwood, at the Department of Zoology, University of Toronto. The exercises were also used and modified from a manual that was produced for " 6 th INTENSIVE IBRO WORKSHOP ON BASIC NEUROSCIENCE" and was held at Korea University, Seoul, South Korea in 1993 (Cooper et al., 1993). The current modifications were required to use equipment common to present day student directed laboratories at various universities. Supported by University of Kentucky, Department of Biology, Office of Undergraduate Studies and College of Arts \& Sciences.

\section{References}

1. Bennett, M. V. L., Barrio, L. C., Bargiello, T. A., Spray, D. C., Hertzberg, E. and Sdez, J. C. Gap junctions: new tools, new answers, new questions. Neuron. 6: 305-320 (1991).

2. Bernardini, G., Peracchia, C., and Peracchia, L.L. Reversible effects of heptanol on gap junction structure and cell-to-cell electrical coupling European Journal of Cell Biology. 34(2):307-312 (1984).

3. Cooper, R.L., Chang, J.J., and Ito, M. A report on the, "SIXTH INTENSIVE IBRO WORKSHOP ON BASIC NEUROSCIENCE", held in July 1993, Seoul, South Korea. Abstracts, Society for Neuroscience 19:116.3 (1993)

4. Cragg, B.G. and Thomas, P.K. The relationship between conduction velocity and the diameter and internodal length of peripheral nerve fibers. Journal of Physiology. 136: 606-614 (1957).

5. Erlanger, J. Gasser, H.S. and Bishop, G.H. The compound nature of the action current of nerves as disclosed by the cathode ray oscillograph. American Journal of Physiology 70: 624-666 (1924).

6. Furshpan, E. J. and Potter, D. D. Transmission at the giant motor synapses of the crayfish. Journal of Physiology. 145(2): 289-325 (1959)

7. Johnston, M. F., Simon, S.A., and Ramrn, F. Interaction of anesthetics with electrical synapses. Nature (Lond.). 286:498-500 (1980).

8. Loewenstein, W. R. Permeability of membrane junctions. Annual NY Academy of Sciences 137: 441-472 (1966).

9. Meda, P., Bruzzone, R., Knodel, S. and Orci, L. Blockage of cell-to-cell communication within pancreatic acini is associated with increased basal release of amylase. Journal of Cell Biology. 103(2):475-483 (1986). 
10. Peracchia, C. Increase in gap junction resistance with acidification in crayfish septate axons is closely related to changes in intracellular calcium but not hydrogen ion concentration Journal of Membrane Biology. 113 (1): 75-92 (1990).

11. Peracchia, C. and Dulhunty, A. F. Low resistance junctions in crayfish: structural changes with functional uncoupling. Journal of Cell Biology. 70: 419-439 (1976).

12. Peracchia, C., Bernardini, G., and Peracchia, L. L. Is calmodulin involved in the regulation of gap junction permeability? Pfügers Arch 399 : 152-154 (1983).

13. Peracchia, C., Lazrak, A. and Peracchia. L. L. Molecular models of channel interaction and gating in gap junctions. In Handbook of Membrane Channels. Molecular and Cellular Physiology. C. Peracchia, editor. Academic Press, San Diego. 361-377 (1994).

14. Spray, D. C., Harris, A. L. and Bennett, M. V. L. Gap junctional conductance is a simple and sensitive function of intracellular pH. Sciences NY 211: 712-715 (1981).

15. Spray, D.C., Harris, L.L. and Bennett, M.V.L. Comparison of $\mathrm{pH}$ and Ca dependence of gap junctional conductance. In Intracellular pH: Its Measurement, Regulation, and Utilization in Cellular Functions, R. Nuccitelli and D. Deamer, eds., pp. 445-461, Alan R. Liss, New York. pp. 445-461 (1982).

16. Spray, D.C., White, R., De Carvalho, C., Harris, A.L. and Bennett, M.L.V. Gating of gap junction channels. Journal of Biophysics 45: 219-230 (1984).

17. Watanabe, A., and Grundfest, H. Impulse propagation at the septal and commissural junctions of crayfish lateral giant axons. Journal of General Physiology 45: 267-308 (1961).

18. Wiersma, C.A.G and Hughes, G.M. On the functional anatomy of neuronal units in the abdominal cord of the crayfish, Procambarus clarkii. Journal of Comparative Neurology 116: 209-228 (1961). 